Behavioural Sciences | Ven Karma Jiga

\title{
Mindfulness-Based Intervention (MBI) provides positive outcomes for those in lower socioeconomic environments
}

The western world today demands a great deal from its inhabitants, both physically and mentally. The prevalence of mental health problems is high, and none more so than those in low socioeconomic status, wis socincial hardship life stress and anxiety. Although trained in both eastern and western based mindfulness techniques, Karma Jiga applies the western secular approach to reach this population. Using an adapted Mindfulness-Based Intervention (MBI), he delivers mindfulness techniques to those physical and mental well-being without charge. The potential benefits to the individual and wider community could prove significant in a world where mental health issues seriously affect 1 in 3 people.

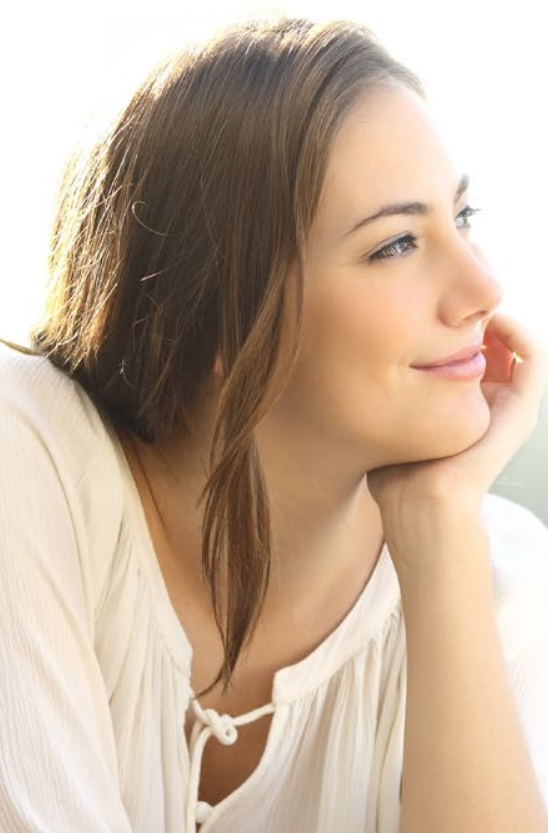

t is no surprise to find that those living

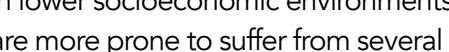
stress-induced physical and mental disorders. Financial stress is often cited disorders. Financial stress is often cited as in westernised autureses, particularly documented that extended periods stress, regardless of source, can inse

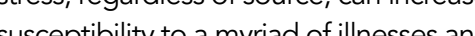
disease, both physical and pychological Most, if not all of us, can relate to experiencing stress at some point in our lives, but unfortunately for some, this can

\section{BRIEF HISTORY OF TREATMENT} APPROACHES

The treatment of stress is now at the forefront of medical and psychological sciences, both striving towards developing the most effective treatments for those who need them. Until relatively recently, the most prevalent approac was pharmacological. However, both pharmacological and nonpharmacological approaches have their aprocts and side effects and ongoing costs of drugs, whereas the psychological approch have high initial costs and a reliance on the will and confidence of the participant to approach their difficulties from a different standpoint. However, for those participating in MBl's from within low socioeconomic environments, it is not play at the same time.

\section{KARMA JIGA AND MINDFULNESS-}

\section{BASED INTERVENTION (MB)}

Many practitioners and ordinary people are now switching their focus towards

The words meditation and mintfuneals. The words medtation and mindfulness are once seemed like a more 'far out' activity is now arguably considered more norma for Westerners. Mindfulness, although it may mean different things to different groups of people, has been defined as the awareness and acceptance of what arises in and around you as it happens; however this requires developing an ability to let go of, and become less mulved with our come to mind.

Typical mindfulness-based interventions, such as Mindfulness-Based Stress Based Cognitive Therapy (MBCT) consist of a 6-8-week programme delivered by trained practitioners. These combine basic Buddhist based meditation practices with a more science-based understanding of stress and wellbeing. The idea is to allow houghts to come and go and let go of the desire to judge or focus on them. By becoming less attached to these thoughts, the negative impact on a person's current mental state is lessened. Reports have yielded signs that these approaches can positively influence physical and . and cortisol levels.

Karma Jiga is trained in both eastern and western mindfulness schools of thought and techniques. He has developed a mindfulness training course aimed at those in low socioeconomic areas called Headroom, and is currently based in Dundee, Scotland. Headroom is a ninesession mindfulness-based intervention (MB), based on the training in MBSR and MBCT he received at Bangor University. He aims to develop mindfulness techniques specific to the problems more commonly faced by those in low socioecon free and open to asbich. Headroom is the red living wage The polengless tha

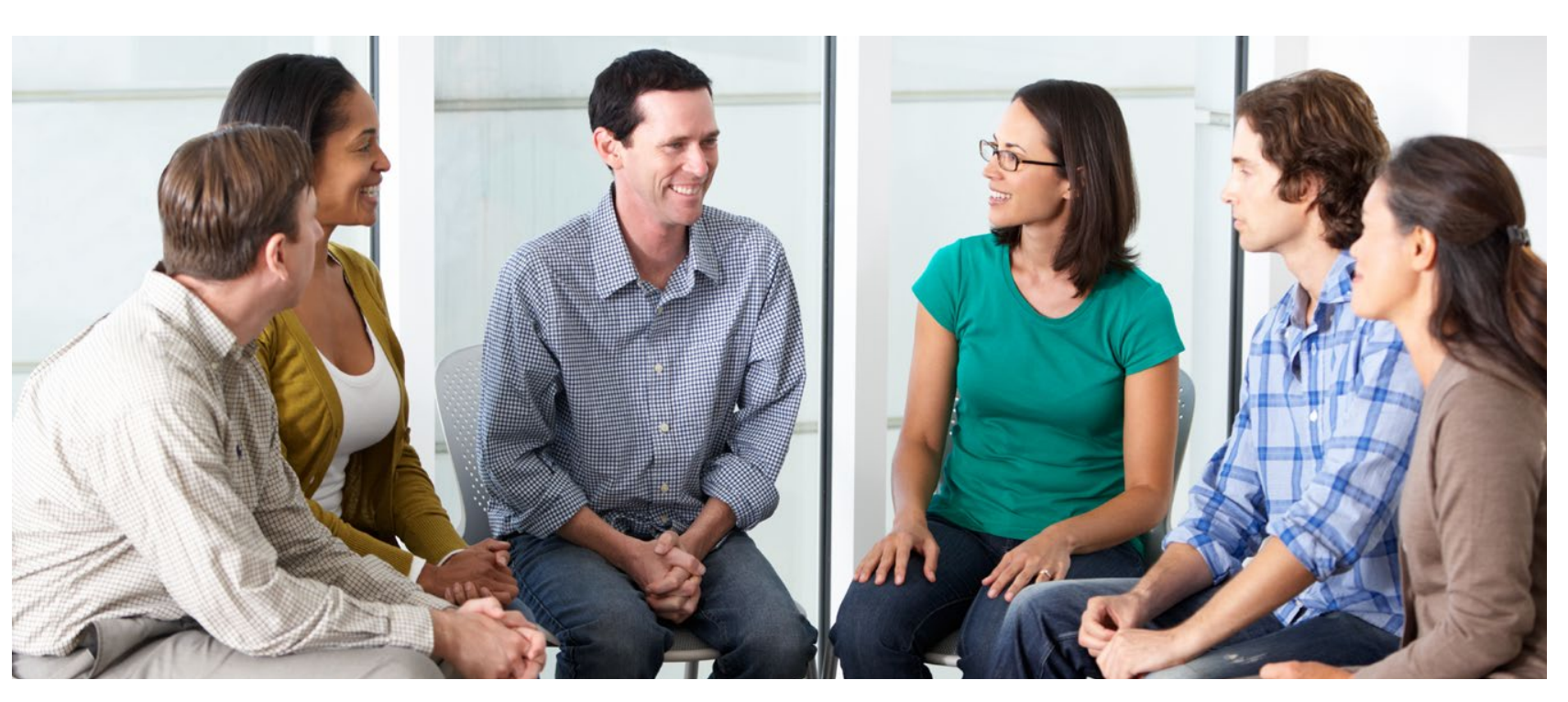

to the community are now becoming known, with participation increasing as completer he curse have sid has fer mel like ther old selves and less ing with circumstances without their control.

\section{THE SCIENCE - DOES IT WORK?} An important measure for any type of

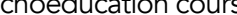
is whether it works and can be verified mpirically. Early research investigating the efficacy of MBls was promising with significant increases in several well-being (Gesures found across twenty studies (Grossman et al, 2004), and whilst som review studies have reported only moderate reductions in anxiety and stress levels (Goyal te al. 2014), the overall tren

Mindfullness has been shown to reverse neurophysiological changes caused by prolonged stress.

and mental health issues encountered by of course completion by individuals.

those in low socioeconomic environments Knowing that high dropout rates were a influences the interpretation of results and makes for a complicated area of study, let alone the fact that genetics may tole in the occurrence of anxiety, stress and depression. Whilst the validity and results of some studies in the mindfulness field have been called in to question, the effects of mindfulness on those in poverty is a unique area of research which needs to be developed.

A primary concern for any MBI programme, especially with low SE ability to produce a viable study Karma Jiga developed a recruitment protocol that would determine the programm initial feasibility. This protocol was effective in that it improved accessibility by providing for participants needs while taking into account participants individual circumstances. Ongoing course evaluation provided information regarding programme feasibility

Participants were 107 referred or selfreferred members of the public living on ROBUST IMPROVEMENTS IN WELL-BEING
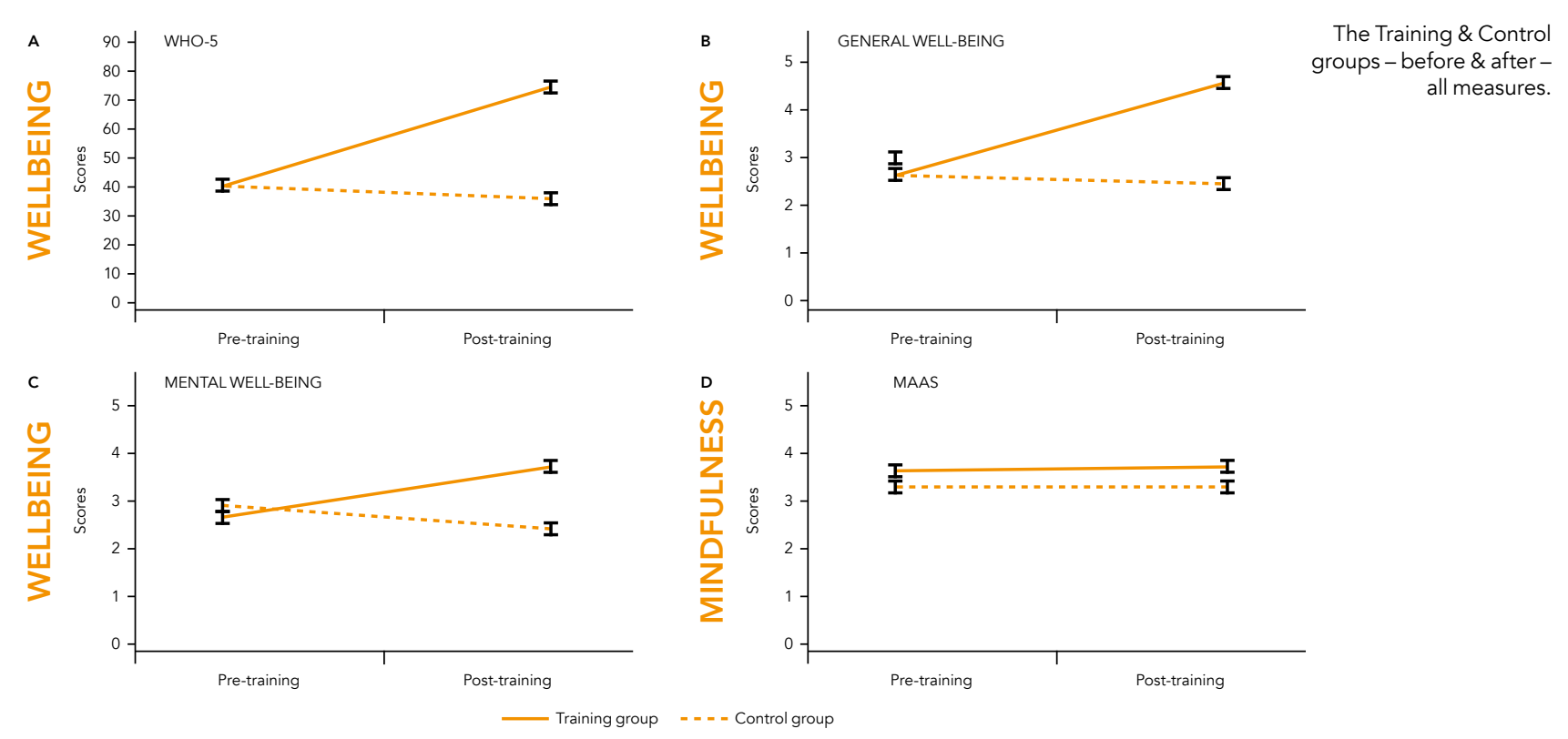


\section{CHANGE IN OUTLOOK}
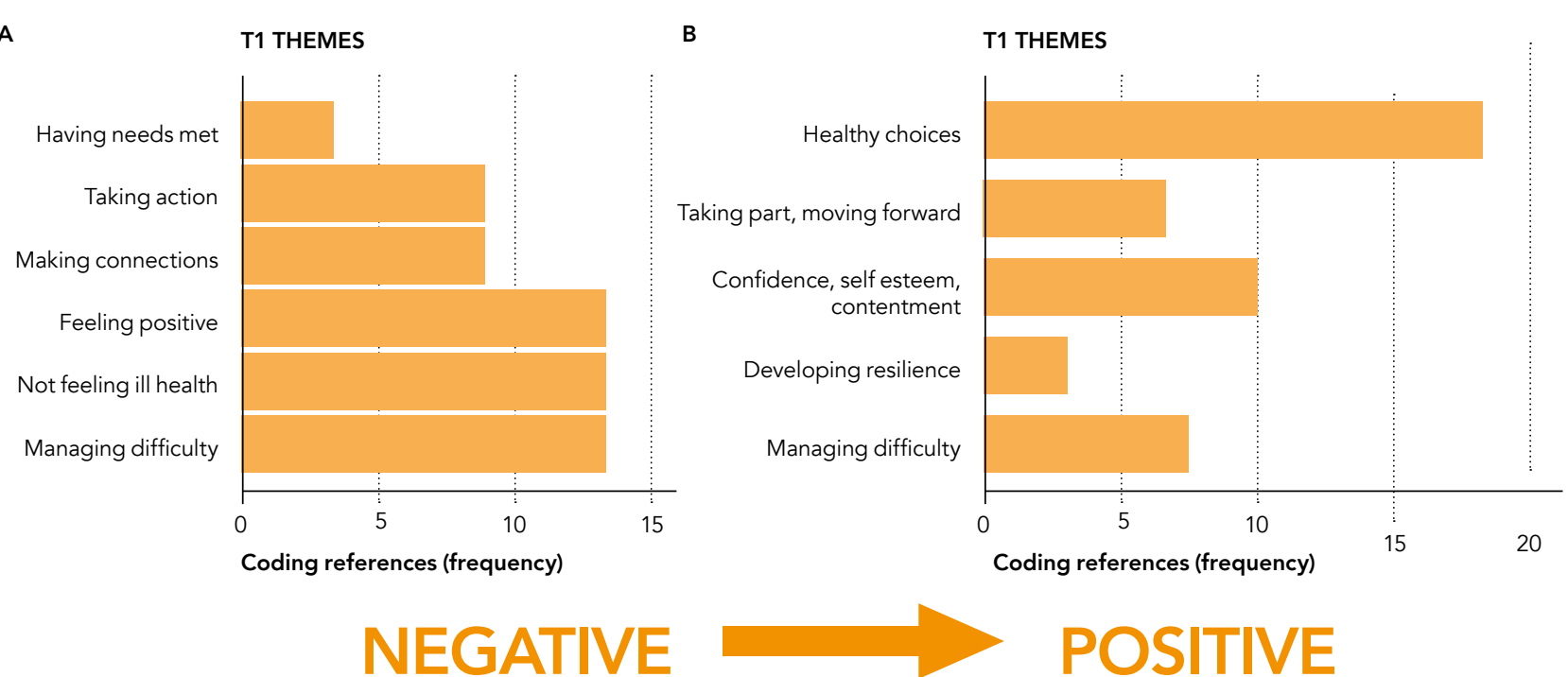

NEGATIVE

Move from 'day to day" reactive decision making, to longer term responsive planning.

\begin{abstract}
low incomes in the most deprived areas of Dundee. However, with post-orientation considerations, the final research group total numbered 40 (20 experimental conditions, 20 control). Participants wer guided through a series of exercises and meditations to improve their sense of awareness and physical and mental wellbeing throughout the 8-week cours. These were assessed through the World Health Organisation (WHO) Well-being Index and Mindfulness Attention and Awareness Scale (MAAS), both widely used and verified in the field. In layman's Weths, his part of the study was gauging whether this adapted MBl course led being whilst assessing attrition rates to stablish the feasibilty of the program further use.

In addition, a thematic and more personal and unique pre and post-course qualitative analysis were carried out to assess how mindfulness practice had affected participants and how they viewed their states of wellbeing before and after the course. Both forms of analysis introduced self-report measures incorporating 5-point
Likert scales. Quantitative measures
\end{abstract}

comparing mean scores between
the control and experimental groups
were conducted. There weren notable
significant differences in scores between
the experimental group completers
and control group completers, with
experimental/training course completers
having significantly higher scores on the
WHO-5. The MAAS measures, however,
showed no significant change and requires
further investigation. Further to this, the
experimental or training group showed
significant positive differences between pre
and post training in the qualitative analysis,
which also gave rise to free expression
through the additition of two open-ended
questions. Thus, positive correlations
between the changes in WHO-5 scores
and General Wellbeing Scores collectively
imply the Headroom MBI typically led to
improvements across well-being measures.
PERSISTING PROBLEMS
OF ATTRITION
The persistent problem of attrition remains,
with the majority of dropouts in Karma
Jiga's and others work in this area due to
reasons beyond the control of the course
provider, or the service user. According
to Karma Jiga's work, a frequent barrier
to course attendance were medication

There is a strong positive correlation between debt and poor physical and mental health. side effects and the time of day he latter being an issue he is currently ttrempting to address and will monitor as the research progresses.

\section{FOOD FOR THOUGHT}

\section{AND FUTURE DIRECTIONS}

Karma Jiga is still providing this incredibly valuable approach to the residents of Dundee and hopefully, this will continue to gain momentum and support from other local authorities. Any ambiguity in the research surrounding mindfulness highlights the need to give this and future research the due attention and opportunity to address any anomalies that anise, as the benefits of mindulness $c a n$ with the effects of phane favourably with little or no negative side effects or ongoing expense. Positive outcomes from mindfulness may not be limited solely to the individual, rather, they have the potential to positively impact the wider community, and in turn the nation. Karma Jiga and his colleagues at Nilupul plan to develop a framework that would allow this programme to be rolled out to other cities, reaching anyone who would benefit. Furthermore, he is looking into how Headroom can be developed into a teaching programme within the further education system to enable peer to peer delivery of the programme within

\section{Behind the Research}

\section{Karma Jiga}

E: karmajiga@nilupul.org T: +0441382872020 W: www.nilupul.org W: www.mindfulnessdundee.co.uk W: www.headroom.nilupul.org 1 w www.facebook.com/nilupulfoundation

\section{Research Objectives}

Karma Jiga and his colleagues at Nilupul Foundation's research is aimed at establishing the feasibility of holding the courses in areas of socioeconomic depression, lowering poverty, and developing mindfulness techniques specific to problems more commonly faced by those in these

\section{Detail}

Nilupul Centre,

51 Reform St,

DD1 1SL

Scotland

Karma Jiga is trained in the Tibetan Buddhist Tradition and modern-day mindfulness. He is a former engineer, award-winning musician, composer, author, artisan, project manager and current $\mathrm{CEO}$ of Nilupul Foundation. His present-day research focus is to provide mental healt solutions for those suffering the effects of poverty.

Funding

Government \& Big Lottery Fund

- Peoples Health Trust

\section{Collaborators}

Conncil

Community Health Team

specialists.

- General Practitioners (GP's)
- Community Support Workers

\section{References}

Grossman, P., Niemann, L., Schmidt, S., \& Walach, H. (2004). Mindfulness-based stress reduction and health benefits'. A metaanalysis. Joumal of Pyychosomatic Research, 57 '. 35-43. https://doi.org/10.1016/50022-3999(03)00573-7.

Goyal, M., Singh, S., Sibinga, E., Gould, N., RowlandSeymour, A., Sharma, R., et al. (2014). Meditation programs

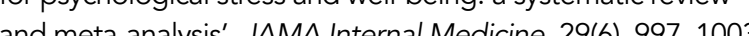
https://doi.org/10.1016/i.biotechadv.2011.08.021.

\section{Personal Response}

\section{What is the main barrier to attendance and completion} of mindfulness courses and
how to get around them?

II At present, the main barrier is cost for the less well off. Mindfulness is more the domain of the middle and more educated class. Even among those who complete a engage meaningfully in the practices betw themselves and difficult to assess. Not everyone is willing to disclose how much or even if they have applied themselves, despite the importance of personal responsibility for individual mental health and wellbeing being emphasised within the course sessions. So reduced cost and inspirational delivery seem

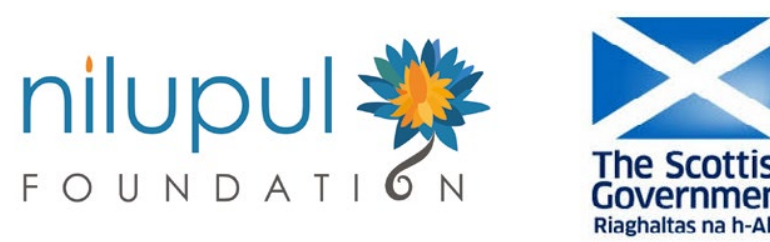

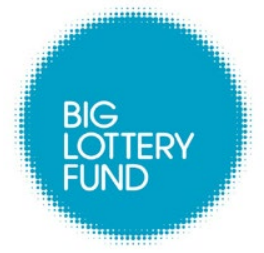

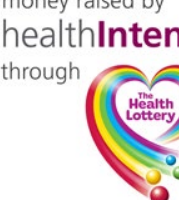

